\title{
Grand Antiprism and Quaternions
}

\author{
Mehmet Koca ${ }^{1}$, Mudhahir Al-Ajmi ${ }^{1}$, Nazife Ozdes Koca ${ }^{1}$ \\ ${ }^{1}$ Department of Physics, College of Science, Sultan Qaboos \\ University, P. O. Box 36, Al-Khoud, 123 Muscat, Sultanate of Oman
}

Email: kocam@squ.edu.om, mudhahir@squ.edu.om, nazife@squ.edu.om

\begin{abstract}
Vertices of the 4-dimensional semi-regular polytope, the grand antiprism and its symmetry group of order 400 are represented in terms of quaternions with unit norm. It follows from the icosian representation of the $E_{8}$ root system which decomposes into two copies of the root system of $H_{4}$. The symmetry of the grand antiprism is a maximal subgroup of the Coxeter group $W\left(H_{4}\right)$. It is the group $A u t\left(H_{2} \oplus H_{2}^{\prime}\right)$ which is constructed in terms of 20 quaternionic roots of the Coxeter diagram $H_{2} \oplus H_{2}^{\prime}$. The root system of $H_{4}$ represented by the binary icosahedral group $I$ of order 120 , constitutes the regular $4 \mathrm{D}$ polytope 600-cell. When its 20 quaternionic vertices corresponding to the roots of the diagram $H_{2} \oplus H_{2}^{\prime}$ are removed from the vertices of the 600-cell the remaining 100 quaternions constitute the vertices of the grand antiprism. We give a detailed analysis of the construction of the cells of the grand antiprism in terms of quaternions. The dual polytope of the grand antiprism has been also constructed.
\end{abstract}

\section{Introduction}

Six regular 4D polytopes and their semi-regular Archimedean polytopes are very interesting because their symmetries $W\left(A_{4}\right), W\left(B_{4}\right), W\left(F_{4}\right)$ and $W\left(H_{4}\right)$ can be represented with the use of finite subgroups of quaternions. There is a close correspondence between the finite subgroups of quaternions and the symmetries of the above polytopes [1]. Some of them occur in high energy physics in model building either as a gauge symmetry like $S U(5)$ [2] or as a little group $S O(9)$ [3] of the M-theory. The Coxeter group $W\left(H_{4}\right)$ arises as the symmetry group of the polytope 600-cell, $\{3,3,5\}$ [4], vertices of which can be represented by 120 quaternions of the binary icosahedral group [5, 6]. The dual polytope 120 -cell, $\{5,3,3\}$ with 600 vertices, can be constructed from 600 -cell in terms of quaternions. Another observation is that they are all nested in the root system of $E_{8}$ represented by icosians [7, 8]. 
The grand antiprism was first constructed in 1965 by Conway and Guy [9] with a computer analysis. An antiprism is defined to be a polyhedron composed of two parallel copies of some particular $n$-sided polygon,connected by an alternating band of triangles. In this paper we study the symmetry group of the grand antiprism and construct its 100 vertices in terms of quaternions. We explicitly show how these vertices form 20 pentagonal antiprisms and 300 tetrahedra. We organize the paper as follows. In Section 2 we briefly discuss the correspondence between the root system of $H_{4}$ and the quaternionic representation of the binary icosahedral group $I$ and decompose it as $120=20+100$ where first 20 quaternions represent the root system of the Coxeter diagram $H_{2} \oplus H_{2}^{\prime}$ and the remaining quaternions are the vertices of the grand antiprism. The symmetry group of the grand antiprism turns out to be the group $\operatorname{Aut}\left(H_{2} \oplus H_{2}^{\prime}\right)$ of order 400 which can be constructed directly from the quaternionic roots of the Coxeter diagram $H_{2} \oplus H_{2}^{\prime}$. A simple technique has been employed in Section 3 for the construction of the cell structures of the grand antiprism. A projection of the grand antiprism into three dimensional space is discussed in Section 4. The dual polytope of the grand antiprism has been constructed in Section 5 where 320 vertices decompose as $320=20+200+100$ under the group $A u t\left(H_{2} \oplus H_{2}^{\prime}\right)$ where 20 vertices are scaled copies of the root system of the Coxeter diagram $H_{2} \oplus H_{2}^{\prime}$ lying on a sphere $S^{3}$ with radius $\frac{1+\sqrt{5}}{2 \sqrt{2}} \approx 1.14$ and 300 vertices lie on an inner sphere $S^{3}$ with a radius of unit length. Conclusion is given in Section 6 and an Appendix lists the decompositions of the vertices of the Archimedian $W\left(H_{4}\right)$ solids in terms of the orbits of $\operatorname{Aut}\left(H_{2} \oplus H_{2}^{\prime}\right)$.

\section{Construction of the root system of $H_{2} \oplus H_{2}^{\prime}$ as the maximal subset of the root system of $H_{4}$ and the vertices of the grand antiprism}

Let $q=q_{0}+q_{i} e_{i},(i=1,2,3)$ be a real quaternion with its conjugate defined by $\bar{q}=q_{0}-q_{i} e_{i}$ where the quaternionic imaginary units satisfy the relations

$$
e_{i} e_{j}=-\delta_{i j}+\varepsilon_{i j k} e_{k},(i, j, k=1,2,3) .
$$

Here $\delta_{i j}$ and $\varepsilon_{i j k}$ are the Kronecker and Levi-Civita symbols respectively and summation over the repeated indices is implicit. Quaternions generate the four dimensional Euclidean space where the quaternionic scalar product is defined by 


$$
(p, q)=\frac{1}{2}(\bar{p} q+\bar{q} p)=\frac{1}{2}(p \bar{q}+q \bar{p}) .
$$

The imaginary quaternionic units $e_{i}$ can be related to the Pauli matrices $\sigma_{i}$ by $e_{i}=-i \sigma_{i}$ and the unit quaternion is represented by $2 \times 2$ unit matrix. This correspondence proves that the group of quaternions is isomorphic to $S U(2)$ which is a double cover of the proper rotation group $S O(3)$. The root system of the Coxeter diagram $H_{4}$ can be represented by the quaternionic elements of the binary icosahedral group $I$ [10] which are given as the set of conjugacy classes in Table 1 . The sets of conjugacy classes are indeed the orbits of the Coxeter group

$$
W\left(H_{3}\right)=\left\{[p, \bar{p}] \oplus[p, \bar{p}]^{*}, \quad p \in I\right\}
$$

where we use the notation [8]

$$
[a, b]: q \rightarrow a q b \text { and }[a, b]^{*}: q \rightarrow a \bar{q} b .
$$

Table 1: Conjugacy classes of the binary icosahedral group $I$ represented by quaternions for the cases of 1 being fixed. (Sets of conjugacy classes

\begin{tabular}{|c|c|c|}
\hline Orders of elements & & $\begin{array}{l}\text { The sets } N(1) \text { of the conjugacy classes } \\
\text { denoted with } N \text { elements }\end{array}$ \\
\hline 1 & 1 & \\
\hline 2 & -1 & \\
\hline 10 & $12(1)_{+}:$ & $\begin{array}{l}\frac{1}{2}\left(\tau \pm e_{1} \pm \sigma e_{3}\right), \frac{1}{2}\left(\tau \pm e_{2} \pm \sigma e_{1}\right) \\
\frac{1}{2}\left(\tau \pm e_{3} \pm \sigma e_{2}\right)\end{array}$ \\
\hline 5 & $12(1)_{-}:$ & $\begin{array}{l}\frac{1}{2}\left(-\tau \pm e_{1} \pm \sigma e_{3}\right), \frac{1}{2}\left(-\tau \pm e_{2} \pm \sigma e_{1}\right) \\
\frac{1}{2}\left(-\tau \pm e_{3} \pm \sigma e_{2}\right)\end{array}$ \\
\hline 10 & $12(1)_{+}^{\prime}:$ & $\begin{array}{l}\frac{1}{2}\left(\sigma \pm e_{1} \pm \tau e_{2}\right), \frac{1}{2}\left(\sigma \pm e_{2} \pm \tau e_{3}\right) \\
\frac{1}{2}\left(\sigma \pm e_{3} \pm \tau e_{1}\right)\end{array}$ \\
\hline 5 & $12(1)_{-}^{\prime}:$ & $\begin{array}{l}\frac{1}{2}\left(-\sigma \pm e_{1} \pm \tau e_{2}\right), \frac{1}{2}\left(-\sigma \pm e_{2} \pm \tau e_{3}\right) \\
\frac{1}{2}\left(-\sigma \pm e_{3} \pm \tau e_{1}\right)\end{array}$ \\
\hline 6 & $20(1)_{+}:$ & $\begin{array}{l}\frac{1}{2}\left(1 \pm e_{1} \pm e_{2} \pm e_{3}\right), \frac{1}{2}\left(1 \pm \tau e_{1} \pm \sigma e_{2}\right) \\
\frac{1}{2}\left(1 \pm \tau e_{2} \pm \sigma e_{3}\right), \frac{1}{2}\left(1 \pm \tau e_{3} \pm \sigma e_{1}\right)\end{array}$ \\
\hline 3 & $20(1)_{-}:$ & $\begin{array}{l}\frac{1}{2}\left(-1 \pm e_{1} \pm e_{2} \pm e_{3}\right), \frac{1}{2}\left(-1 \pm \tau e_{1} \pm \sigma e_{2}\right) \\
\frac{1}{2}\left(-1 \pm \tau e_{2} \pm \sigma e_{3}\right), \frac{1}{2}\left(-1 \pm \tau e_{3} \pm \sigma e_{1}\right)\end{array}$ \\
\hline 4 & $30(1):$ & $\begin{array}{l} \pm e_{1}, \pm e_{2}, \pm e_{3}, \frac{1}{2}\left( \pm \sigma e_{1} \pm \tau e_{2} \pm e_{3}\right) \\
\frac{1}{2}\left( \pm \sigma e_{2} \pm \tau e_{3} \pm e_{1}\right), \frac{1}{2}\left( \pm \sigma e_{3} \pm \tau e_{1} \pm e_{2}\right)\end{array}$ \\
\hline
\end{tabular}
of $N$ elements fixing the elements $q$ are denoted as $N(q)$ where additional subscripts are used to distinguish different sets of the same type.) 
In Table $1 \tau=\frac{1+\sqrt{5}}{2}, \quad \sigma=\frac{1-\sqrt{5}}{2}$ and they satisfy the relations $\tau^{2}=\tau+$ $1, \sigma^{2}=\sigma+1, \tau+\sigma=1, \tau \sigma=-1$. The notation \# (1) denote the set of elements of the conjugacy classes which also represent the orbits of the Coxeter group $W\left(H_{3}\right)$ fixing the element 1 .

Let $b=\frac{1}{2}\left(\tau+\sigma e_{1}+e_{2}\right) \in 12(1)_{+}$and $b^{5}=-1$ represent the two simple roots of the Coxeter group $\mathrm{H}_{2}$ with unit norm. Then the quaternions $e_{3} b=$ $\frac{1}{2}\left(-e_{1}+\sigma e_{2}+\tau e_{3}\right)$ and $e_{3} b^{5}=-e_{3}$ represent the simple roots of the Coxeter diagram $H_{2}^{\prime}$ orthogonal to the roots of $H_{2}$. The Coxeter diagram of $H_{2} \oplus H_{2}^{\prime}$ with its simple roots are depicted in Figure 1.

The reflection generators at these simple roots are given by [11]

$$
[b,-b]^{*}, \quad[1,-1]^{*}, \quad\left[e_{3} b,-e_{3} b\right]^{*}, \quad\left[e_{3},-e_{3}\right]^{*} .
$$

These generators will lead to the following 20 quaternionic roots of the Coxeter diagram $H_{2} \oplus H_{2}^{\prime}$

$$
H_{2} \oplus H_{2}^{\prime}=\left\{b^{m}, \quad \mathrm{e}_{3} b^{m}\right\},(m=0,1, \ldots, 9) .
$$

This set of quaternions form a maximal subgroup, the dicyclic group of order 20, in the binary icosahedral group $I$ [4]. The generators in (5) generate the group $W\left(H_{2}\right) \times W\left(H_{2}^{\prime}\right)$, each of which, is a dihedral group of order 10 . It is more appropriate to represent each dihedral group with one rotation generator and one reflection generator which can be written as

$$
W\left(H_{2}\right)=\left\{[b, b],[1,-1]^{*}\right\} ; \quad W\left(H_{2}^{\prime}\right)=\left\{[\bar{b}, b],\left[e_{3},-e_{3}\right]^{*}\right\} .
$$

The direct product group is of order 100. One may simply note that the generator $\left[e_{3}, 1\right]$ or $\left[1, e_{3}\right]$ permutes the simple roots of $H_{2} \oplus H_{2}^{\prime}$. Actually these generators leave the Cartan matrix of the diagram $H_{2} \oplus H_{2}^{\prime}$ invariant. Without loss of generality we take the generator $\left[e_{3}, 1\right]$ as the new generator which generates the cyclic group $C_{4}$ of order 4 . The group $C_{4}$ is the normalizer of the group $W\left(H_{2}\right) \times W\left(H_{2}^{\prime}\right)$ in the Coxeter group $W\left(H_{4}\right)$. An extension of the group $W\left(H_{2}\right) \times W\left(H_{2}^{\prime}\right)$ by the group $C_{4}$ is a semi-direct product of the two groups which is the group

$$
\begin{gathered}
\operatorname{Aut}\left(H_{2} \oplus H_{2}^{\prime}\right) \approx\left\{W\left(H_{2}\right) \times W\left(H_{2}^{\prime}\right)\right\}: C_{4} \\
\alpha_{1}=b \quad \alpha_{2}=-1 \quad \alpha_{1}^{\prime}=e_{3} b \quad \alpha_{2}^{\prime}=-e_{3}
\end{gathered}
$$

Figure 1: Coxeter diagram of $H_{2} \oplus H_{2}^{\prime}$ with quaternionic simple roots. 
of order 400 and is one of the five maximal subgroups of the Coxeter group $W\left(H_{4}\right)[11]$.

The group elements now can be written more concisely as

$$
\operatorname{Aut}\left(H_{2} \oplus H_{2}^{\prime}\right)=\left\{[p, q] \oplus[p, q]^{*} ; \quad p, q \in\left(H_{2} \oplus H_{2}^{\prime}\right)\right\} .
$$

It is represented by the pairs of quaternions taking values from the root system of the Coxeter diagram $H_{2} \oplus H_{2}^{\prime}$. This is the quaternionic representation of the ionic diminished Coxeter group $\left[10,2^{+}, 10\right]$ [6].

\section{Construction of vertices of the grand an- tiprism}

The set of roots of the Coxeter graph $H_{2} \oplus H_{2}^{\prime}$ represents the vertices of two decagons in two orthogonal planes sharing only the origin as the common point. For a better clarification let us define a new set of quaternionic units

$$
1^{\prime}=1, \quad e_{1}^{\prime}=\frac{\sigma e_{1}+e_{2}}{\sqrt{2+\sigma}}, \quad e_{2}^{\prime}=e_{3} e_{1}^{\prime}=\frac{-e_{1}+\sigma e_{2}}{\sqrt{2+\sigma}}, e_{3}^{\prime}=e_{3} .
$$

Then the set of roots in (마) can be written as

$$
\left\{\exp \left(\frac{\pi}{5} e_{1}^{\prime}\right)^{m}, \quad e_{3} \exp \left(\frac{\pi}{5} e_{1}^{\prime}\right)^{m}\right\}, \quad(m=0,1, \ldots, 9) .
$$

This indicates that the roots of the first $\mathrm{H}_{2}$ and the origin define a plane, say plane $\mathbf{A}$, generated by the unit vectors $1, e_{1}^{\prime}$ and the second set of roots with the origin define the plane $\mathbf{B}$, generated by the unit vectors $e_{2}^{\prime}$ and $e_{3}$. The group element $[b, b]$ acts as a rotation of order 5 in the plane $\mathbf{A}$ and the element $[\bar{b}, b]$ acts as a rotation of order 5 in the plane $\mathbf{B}$. In what follows we prove that the remaining set of 100 quaternions obtained by deleting those quaternions in (6) from the set $I$, can be written compactly as vertices of the grand antiprism (GA):

$$
\mathrm{GA}=\left\{b^{m} c b^{n}, b^{m} e_{3} c b^{n}\right\}, \quad(m, n=0,1, \ldots, 9) .
$$

Here $c=\frac{1}{2}\left(\tau-\sigma e_{1}+e_{2}\right) \in 12(1)_{+}$is one of those nearest quaternions to $b$ in $I$. In reference [10] we showed that the sets of quaternions in Table 1 represent the vertices of some polyhedra possessing the icosahedral symmetry $W\left(H_{3}\right)$. Each set of 12 elements represents an icosahedron, each set of 20 elements represents a dodecahedron and the set with 30 elements represents an icosidodecahedron. The unit quaternions \pm 1 represent the poles of the 
sphere $S^{3}$. It is clear that these polyhedra have the vertices at the intersections of the parallel hyperplanes orthogonal to the unit quaternion 1 with the sphere $S^{3}$. For example, the set $12_{+}(1)$ can be plotted in a space where the imaginary unit quaternions represent the ordinary $x, y, z$ axes. This means we are in the space represented by the intersection of the sphere $S^{3}$ with the hyperplane orthogonal to the unit quaternion 1 and at a distance $\frac{\tau}{2}$ from the origin.

In order to understand the 600 -cell, the set $12(1)_{+}$plays the most important role. The quaternions in the set $12(1)_{+}$constitute the vertices of an icosahedron with the shortest edge length $-\sigma$. They can be decomposed into 20 sets of triads [10] where each triad represents an equilateral triangle. The edge length between the unit quaternion 1 and any one of those quaternions in the set $12(1)_{+}$is also $-\sigma$ so that the quaternion 1 and the quaternions of the set $12(1)_{+}$constitute 20 tetrahedra with a common vertex represented by 1 . Table 1 is just one decomposition out of 120 different decompositions where any quaternion $q \in I$ can be chosen instead of 1 . This is because the group $W\left(H_{3}\right)$ can be embedded in 120 different ways in the group $W\left(H_{4}\right)$. In each case the group $W\left(H_{3}\right)$ leaves one quaternion, $q$ say, invariant which can be represented by the conjugate group $W\left(H_{3}\right)^{q}=\left\{[I, \bar{q} \bar{I} q] \oplus[I, q \bar{I} q]^{*}\right\}[10]$. The 20 tetrahedra having the quaternion $q$ as a common vertex would be represented by the set of quaternions $q$ and $\mathrm{q}\left(12(1)_{+}\right)=\left(12(1)_{+}\right) q=12(q)_{+}$. This proves that the 600 -cell consists of $\frac{20 \times 120}{4}=600$ tetrahedra.

The set $12(1)_{+}$can be written in terms of the quaternions $b$ and $c$ as follows:

$$
\begin{aligned}
& \text { b } \\
& c \quad \bar{b} c b \quad \bar{b}^{2} c b^{2} \quad b^{2} c \bar{b}^{2} \quad b c \bar{b} \\
& c \bar{b} \quad \begin{array}{cccc}
\bar{b} c & \bar{b}^{2} c b & -b^{2} c b^{2} & b c \bar{b}^{2} \\
\bar{b} & &
\end{array}
\end{aligned}
$$

Indeed the third line is the set of five quaternions $\left(\bar{b}^{m} \bar{c} b^{m}, m=\{0,1,2,3,4\}\right)$ obtained by rotating the quaternion $\bar{c}$ around the quaternion $b$. This assertion can be checked through the relations

$$
b^{2} c b^{2}=-\bar{c}, \quad \bar{b} c b=c b \bar{c}, \quad \bar{c} b c=b c \bar{b}, \quad e_{3} b=\bar{b} e_{3}, \quad e_{3} c=\bar{c} e_{3} .
$$

As we stated earlier they are the vertices of an icosahedron when plotted in the ordinary 3D space represented by $x, y, z$ as depicted in Figure 2.

If one imagines that the quaternions $b$ and $\bar{b}$ represent the top and the bottom vertices of the icosahedron in (13) then those quaternions in the second line constitute the vertices of a pentagon nearest to the quaternion $b$ and those in 


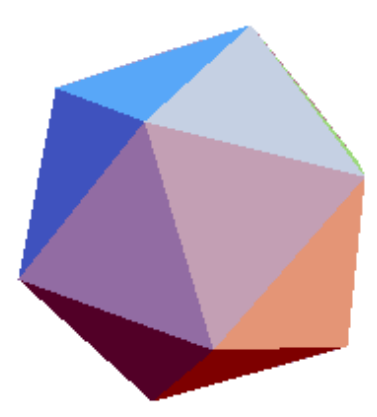

Figure 2: The icosahedron of the quaternions of Eq.13

the third line represent the bottom pentagonal plane nearest to the quaternion $\bar{b}$. One can easily show that the quaternions representing the edges of the pentagons in both top and bottom planes can be written as follows

$$
-\sigma\left(\alpha e_{2}^{\prime}+\beta e_{3}\right), \alpha^{2}+\beta^{2}=1 .
$$

Indeed these two planes are parallel to the plane $\mathbf{B}$ which is orthogonal to the plane $\mathbf{A}$ generated by 1 and $e_{1}^{\prime}$. Since $b$ and $\bar{b}$ are removed from the set of quaternions in (13) what remains is the pentagonal antiprism with 10 vertices which is depicted in Figure 3.

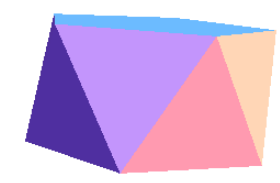

Figure 3: Pentagonal antiprism represented by the quaternionic vertices $\left(\bar{b}^{m} c b^{m}, \bar{b}^{m} \bar{c} b^{m}\right),(m=0,1,2,3,4)$.

There is now a simple mechanism to generate 10 pentagonal antiprisms which form a ring. Before proceeding further we note that all quaternions on the second line of (13) are obtained from the vertex $c$ by a rotation in the plane $\mathbf{B}$ by an angle $72^{0}$. Similarly the quaternions on the third line of (13) are obtained by a $72^{0}$ rotation of $\bar{c}$ in the plane parallel to the plane $\mathbf{B}$.

This shows that the edge length of the pentagon is $-\sigma$.

Now we discuss how to obtain those 50 quaternions constituting the first ring of 10 pentagonal anti-prisms. Let us consider the diagram in Figure 4 


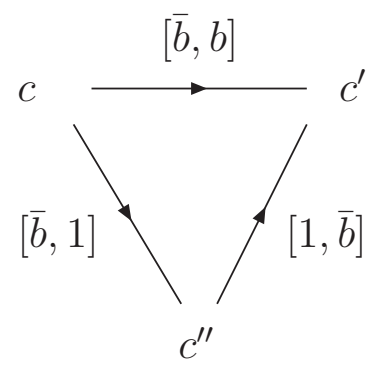

Figure 4: Diagrammatic representation of some of the group elements of $\operatorname{Aut}\left(H_{2} \oplus H_{2}^{\prime}\right)$

This shows that the group element $[\bar{b}, b]$ rotates the quaternions by an angle $72^{0}$ in the plane $\mathbf{B}$, in other words, in the ten planes parallel to $\mathbf{B}$. The group element $[\bar{b}, 1]$ transforms a given quaternion in the 'upper plane' to a quaternion in the 'lower plane' which is already rotated by $36^{0}$ degrees with respect to the 'upper plane'. The group element $[1, b]$ lifts one element from the bottom plane to the upper plane after a $36^{0}$ rotation. It is interesting to note that just as the sum of these vectors $\left(c^{\prime \prime}-c\right)+\left(c^{\prime}-c^{\prime \prime}\right)=c^{\prime}-$ $c$, the group elements satisfy a similar relation, namely, $[\bar{b}, 1][1, b]=[\bar{b}, b]$. Inverses of these group elements reverse the directions of the arrows. The quaternions $c, \quad c^{\prime}=\bar{b} c b, \quad c^{\prime \prime}=\bar{b} c$ form an equilateral triangle. Applying $[1, b]$ ten times on any quaternion will bring it back to the original quaternion so that they define a decagon. Similarly the action of $[\bar{b}, 1]$ on any quaternion ten times will generate another plane of decagon. It is clear that we obtain the following ring of ten pentagonal antiprisms provided the quaternions $b^{m},(m=0,1, \ldots, 9)$ are removed from the set $I$.

Figure 5 indicates that when starting with the quaternion $c$ we obtain the set of 50 quaternions

$$
R_{1}=\left\{b^{m} c b^{n}\right\}, \quad(m, n=0,1, \ldots, 9) .
$$

It is clear from Figure 5 that the vertices of two pentagonal antiprisms in which the quaternion $c$ is a common vertex can be written as follows:

$$
\begin{aligned}
c b, \quad \bar{b} c b^{2}, \quad-\bar{b}^{2} c \bar{b}^{2}, \quad b^{2} c \bar{b}, & b c \\
c, \quad \bar{b} c b, \quad \bar{b}^{2} c b^{2}, \quad b^{2} c \bar{b}^{2}, \quad b c \bar{b} & \\
\bar{b} c, \quad \bar{b}^{2} c b, \quad-b^{2} c b^{2}, \quad b c \bar{b}^{2}, \quad c \bar{b} &
\end{aligned}
$$

Note that the quaternions at the upper two lines can be obtained from those at the lower two lines by a right multiplication by the quaternion $b$. In other 


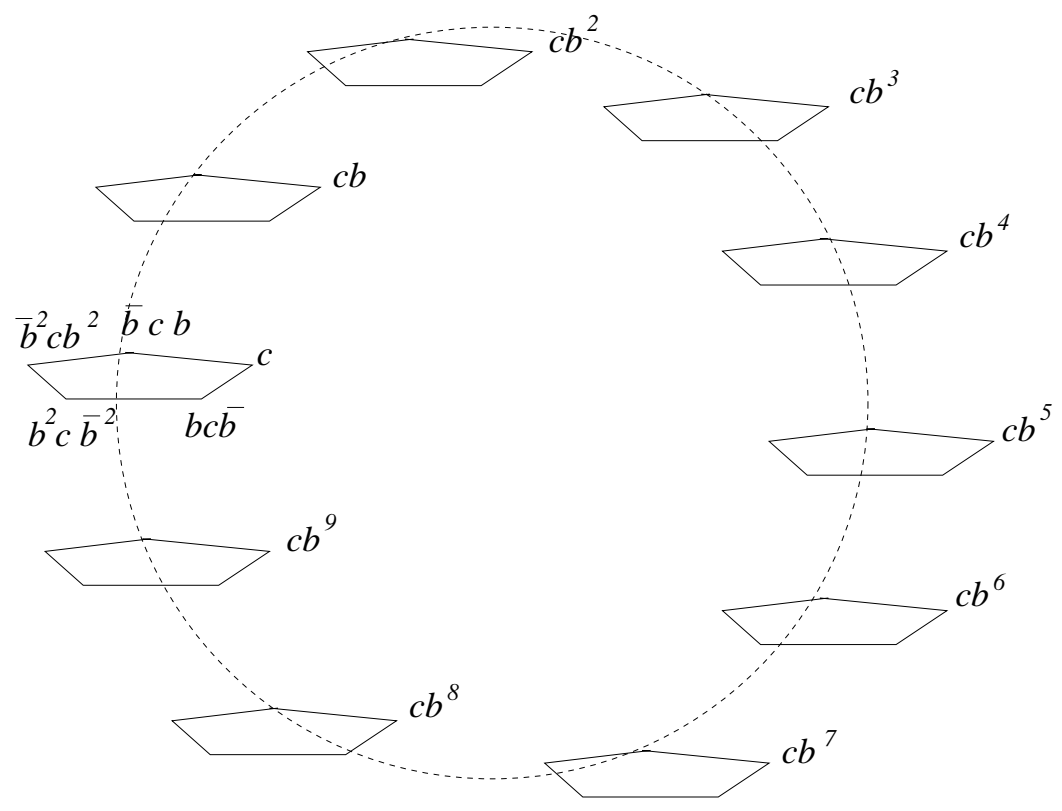

Figure 5: One ring of pentagonal antiprism. The quaternions at the vertices of the pentagons are obtained by $\frac{2 \pi}{5}$ rotations around the vertex $b$.

words, the set of quaternions in the upper two lines are the elements of the set $12_{+}(b)$ from which 1 and $b^{2}$ are deleted. They constitute the pentagonal antiprism in the space of intersection of the hyperplane orthogonal to the quaternion $b$ and the sphere $S^{3}$. We note that the quaternions at the bottom line are the quaternionic conjugates of the quaternions at the middle line. Therefore the center of the lower pentagonal antiprism is at the point $\frac{\tau}{2} 1$ and the center of the upper pentagonal antiprism is at the quaternion $\frac{\tau}{2} b$. If we keep multiplying the quaternions representing a given pentagonal antiprism by $b$ we create other quaternionic vertices. The next layer of vertices are the set of $12_{+}\left(b^{2}\right)$ with the omission of $b$ and $b^{3}$ where the center is represented by $\frac{\tau}{2} b^{2}$. We have altogether ten pentagonal antiprisms in this ring represented by the sets $12_{+}\left(b^{m}\right)$, where the vertices $b^{m}, m=0,1, \ldots, 9$ are deleted from the sets and the centers of the pentagonal antiprisms are represented by ten quaternions $\frac{\tau}{2} b^{m}$. We note that the action of the element $[1, b]$ corresponds to the rotation of the hyperplane whose intersection with the sphere $S^{3}$ is a pentagonal antiprism.

When we act by the group element $\left[e_{3}, 1\right]$ on the set of quaternions in the diagram of Figure 4 and find the conjugate elements of the group elements we obtain the diagram in Figure 6.

A similar consideration will lead to the set of quaternions 


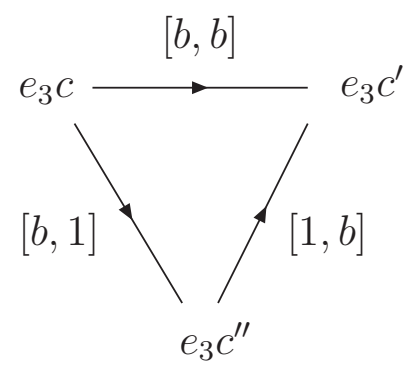

Figure 6: Diagrammatic representation of the action of some group elements on the set of vertices of the second ring of the pentagonal anti-prisms.

$$
R_{2}=\left\{b^{m} e_{3} c b^{n}\right\}, \quad(m, n=0,1, \ldots, 9)
$$

which represents the second ring of vertices of ten pentagonal antiprisms similar to those depicted in Figure 5. The two sets of rings satisfy the relations

$$
e_{3} R_{1}=R_{1} e_{3}=R_{2}, \quad e_{3} R_{2}=R_{2} e_{3}=R_{1} .
$$

It is clear from these discussions that the centers of the pentagonal antiprisms in both rings are represented by the set of 20 quaternions

$$
\frac{\tau}{2} b^{m}, \quad \frac{\tau}{2} e_{3} b^{m}, \quad(m=0,1, \ldots, 9) .
$$

The grand antiprism has 300 tetrahedral cells, details of which we discuss below. In order to understand the cell structure of a polytope one should check first the cell structures surrounding a given vertex, say, vertex $c$. We have already seen that the vertex $c$ is surrounded by two pentagonal antiprisms illustrated in Eq. (17). To understand the nature of the tetrahedra linked to the vertex $c$ we look at the set of quaternions $12_{+}(c)=12_{+}(1) c=c\left(12_{+}(1)\right)$ which can be written as follows:

$$
\begin{gathered}
c^{2}, \quad c \bar{b} c b, \quad c \bar{b}^{2} c b^{2}, \quad b, \quad c b c \bar{b} \\
c \bar{b} c, \quad c \bar{b}^{2} c b, \quad \frac{1,}{c \bar{b}} \quad c b c \bar{b}^{2}, \quad c^{2} \bar{b}
\end{gathered}
$$

We note that we should remove two quaternions 1 and $b$ from the set of (21) since they are the roots of $\mathrm{H}_{2}$. It is easy to see that the remaining 10 vertices form 12 equilateral triangles. When they are connected to the vertex $c$ we 
obtain 12 tetrahedra. This proves that the vertex $c$ is surrounded by two pentagonal antiprisms and 12 tetrahedra. Let us write those 10 quaternions explicitly

$$
\begin{array}{ll}
q_{1}=\frac{1}{2}\left(1+e_{1}+e_{2}-e_{3}\right), & q_{2}=\frac{1}{2}\left(1+\tau e_{2}-\sigma e_{3}\right), \\
q_{3}=\frac{1}{2}\left(\tau+e_{1}-\sigma e_{3}\right), & q_{4}=\frac{1}{2}\left(1+\tau e_{1}-\sigma e_{2}\right), \\
q_{5}=\frac{1}{2}\left(\tau-\sigma e_{2}+e_{3}\right), & q_{6}=\frac{1}{2}\left(-\sigma+e_{1}+\tau e_{2}\right), \\
q_{7}=\frac{1}{2}\left(\tau+e_{1}+\sigma e_{3}\right), & q_{8}=\frac{1}{2}\left(\tau-\sigma e_{2}-e_{3}\right), \\
q_{9}=\frac{1}{2}\left(1+e_{1}+e_{2}+e_{3}\right), & q_{10}=\frac{1}{2}\left(1+\tau e_{2}+\sigma e_{3}\right)
\end{array}
$$

They belong to the following sets of rings of pentagonal antiprisms

$$
\left(c, q_{2}, q_{3}, q_{5}, q_{7}, q_{8}, q_{10}\right) \in R_{1} ; \quad\left(q_{1}, q_{4}, q_{6}, q_{9}\right) \in R_{2} .
$$

The 12 tetrahedra can be classified as follows:

Four tetrahedra:

$$
\left(c, q_{2} ; q_{6}, q_{9}\right), \quad\left(c, q_{3} ; q_{4}, q_{9}\right), \quad\left(c, q_{7} ; q_{1}, q_{4}\right), \quad\left(c, q_{10} ; q_{1}, q_{9}\right)
$$

Six tetrahedra:

$$
\begin{aligned}
& \left(c, q_{2}, q_{5} ; q_{9}\right),\left(c, q_{2}, q_{10} ; q_{6}\right),\left(c, q_{3}, q_{5} ; q_{9}\right),\left(c, q_{3}, q_{7} ; q_{4}\right), \\
& \left(c, q_{7}, q_{8} ; q_{1}\right),\left(c, q_{8}, q_{10} ; q_{1}\right)
\end{aligned}
$$

Two tetrahedra:

$$
\left(c ; q_{1}, q_{4}, q_{6}\right), \quad\left(c ; q_{4}, q_{6}, q_{9}\right) .
$$

It is clear from these considerations that the first four tetrahedra in (24) have vertex structures such that two vertices are on the ring $R_{1}$ and two vertices are on the ring $R_{2}$. The next six tetrahedra in (25) have vertex structures such that three vertices forming an equilateral triangle on the ring $R_{1}$ and one vertex on the ring $R_{2}$. Similarly the last two tetrahedra in (26) have the following structure: one vertex on the ring $R_{1}$ and three vertices on the ring $R_{2}$. When we consider the tetrahedral structures formed by the quaternion $e_{3} c$ and the set of quaternions $12_{+}\left(e_{3} c\right)$, the above structures will be reversed because multiplication by the quaternion $e_{3}$ reverses the sets of rings $e_{3}: \quad R_{1} \leftrightarrow R_{2}$. When we consider the 24 tetrahedra together we see that they split as $24=8+8+8$. Since for one vertex of the grand antiprism we have 12 tetrahedra and each tetrahedron has 4 vertices then the grand antiprism has $\frac{12 \times 100}{4}=300$ tetrahedra which split as $300=100+200$. This shows that 100 tetrahedra have the structure such that two vertices are on the ring $R_{1}$ and two vertices are on the ring $R_{2}$. The 200 tetrahedra have the structure such that each of 100 tetrahedra has three vertices on the ring $R_{1}$ and the fourth vertex on the ring $R_{2}$ and each of the next 100 tetrahedra 
has one vertex on the ring $R_{1}$ and three vertices on the ring $R_{2}$. The ten quaternions in (22) constitute the vertices of the vertex figure of the grand antiprism (see Figure 7) as they are the nearest vertices to the vertex $c$. They are on the sphere $S^{2}$ determined as the intersection of the sphere $S^{3}$ and the hyperplane orthogonal to the quaternion $c$. To plot the vertices in Figure 7 in $3 \mathrm{D}$ we introduce a new set of orthogonal basis of quaternionions defined by

$$
\begin{array}{ll}
d_{0}=c=\frac{1}{2}\left(\tau-\sigma e_{1}+e_{2}\right), & d_{1}=e_{1} c=\frac{1}{2}\left(\sigma+\tau e_{1}+e_{3}\right), \\
d_{2}=e_{2} c=\frac{1}{2}\left(-1+\tau e_{2}+\sigma e_{3}\right), & d_{3}=e_{3} c=\frac{1}{2}\left(-e_{1}-\sigma e_{2}+\tau e_{3}\right) .
\end{array}
$$

In the new basis a quaternion $q$ would read

$$
q=q_{0} d_{0}+q_{1} d_{1}+q_{2} d_{2}+q_{3} d_{3} \equiv\left(q_{0}, q_{1}, q_{2}, q_{3}\right)
$$

and the 10 quaternions above all have first components equal to $\frac{\tau}{2}$. If their first components are deleted and an overall factor $\frac{1}{2}$ is omitted then they can be written as

$$
(0, \pm \sigma, \pm 1), \quad(-\sigma, \pm 1,0), \quad(\sigma, 1,0), \quad(1,0, \pm \sigma), \quad(-1,0, \sigma) .
$$

These are the vertices of the solid called dissected icosahedron shown in Figure 7 .

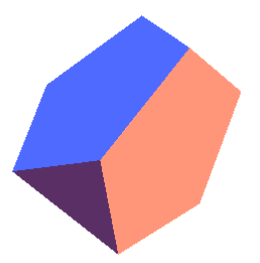

Figure 7: Dissected icosahedron (Vertex figure of the grand antiprism)

The quaternions 1 and $b$ which were deleted can be represented by $(-1,0, \sigma)$, $(\sigma,-1,0)$ respectively in the new basis. With the inclusion of these two vertices to the vertices in (28) one would obtain an icosahedron [12].

Before we end this section we determine the symmetry of the vertex figure. The subgroup of the group $A u t\left(H_{2} \oplus H_{2}^{\prime}\right)$ fixing the quaternion $c$, in other words, leaving the hyperplane orthogonal to $c$ invariant is the symmetry of 
the vertex figure. Therefore it suffices to determine the subgroup which leaves the quaternion $c$ invariant. One can check that it is the group generated by two commuting group elements $\left[e_{3},-e_{3}\right]^{*}$ and $\left[\bar{b}^{2},-\bar{b}^{2}\right]^{*}$. So it is the Klein's four-group $C_{2} \times C_{2}$ of order 4 . The vertices of the dissected icosahedron which are left invariant under the action of the group $C_{2} \times C_{2}$ are given as sets of quaternions in the brackets as follows:

$$
\left(q_{1}, q_{9}\right),\left(q_{2}, q_{3}, q_{7}, q_{10}\right),\left(q_{4}, q_{6}\right),\left(q_{5}, q_{8}\right)
$$

\section{Projection of the grand antiprism into 3D space}

Let us consider the intersection of the hyperplanes orthogonal to the unit quaternion 1 with the three dimensional sphere $S^{3}$ of unit radius consisting of 120 vertices of the 600-cell displayed in Table 1 . When the set of vectors $b^{m}, \mathrm{e}_{3} b^{m}(m=0,1,2, \ldots, 9)$ are removed from the set of quaternions of Table 1, we obtain several pentagonal antiprisms some of which are not regular. The regular pentagonal prisms follow from the sets $12_{ \pm}(1)$ and $12_{ \pm}^{\prime}(1)$ when $\pm b, \pm \bar{b}, \pm b^{2}, \pm \bar{b}^{2}$ are removed from the sets. The vertices of four pentagonal antiprisms can be compactly written as;

$$
\begin{aligned}
& \left(\bar{b}^{m} c b^{m}, \bar{b}^{m} \bar{c} b^{m}\right), \quad\left(-\bar{b}^{m} c b^{m},-\bar{b}^{m} \bar{c} b^{m}\right), \\
& \left(\bar{b}^{m} c^{2} b^{m}, \bar{b}^{m} \bar{c}^{2} b^{m}\right), \quad\left(-\bar{b}^{m} c^{2} b^{m},-\bar{b}^{m} \bar{c}^{2} b^{m}\right), \quad(m=0,1,2,3,4) .
\end{aligned}
$$

They are in the intersections of the sphere $S^{3}$ with the parallel hyperplanes orthogonal to the unit quaternion 1 and with real coordinates at the distances $\pm \frac{\tau}{2}$ and $\pm \frac{\sigma}{2}$. One of these pentagonal antiprism has been depicted in Figure 3 . Similarly, at distances $\pm \frac{1}{2}$ from the origin along the quaternion 1 we have two dodecahedra, namely the sets $20_{+}$and $20_{-}$one of which is depicted in Figure 8.

One of the sets, say, $20_{+}$now decomposes into two sets as $20_{+}=10_{+}+10_{+}^{\prime}$ under a subgroup, of order 20, of the group $\operatorname{Aut}\left(H_{2} \oplus H_{2}^{\prime}\right)$. It is the group $C_{2} \times W\left(H_{2}^{\prime}\right)$ which leaves the unit quaternion 1 invariant. The generators of the group can be written as $C_{2} \times W\left(H_{2}^{\prime}\right)=\left\{[1,1]^{*},[\bar{b}, b],\left[e_{3},-e_{3}\right]^{*}\right\}$. The other set $20_{-}$has a similar decomposition. Each set of quaternions $10_{+}$and $10_{+}^{\prime}$ can be written as 


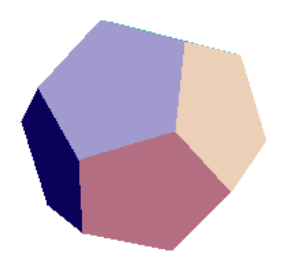

Figure 8: Dodecahedron obtained from the projection of the grand antiprism

$$
\begin{aligned}
10_{+}= & \left\{\frac{1}{2}\left(1+e_{1}+e_{2}+e_{3}\right), \frac{1}{2}\left(1-e_{1}-e_{2}-e_{3}\right),\right. \\
& \frac{1}{2}\left(1-e_{1}-e_{2}+e_{3}\right), \frac{1}{2}\left(1+e_{1}+e_{2}-e_{3}\right), \\
& \left.\frac{1}{2}\left(1 \pm \sigma e_{1} \pm \tau e_{3}\right), \frac{1}{2}\left(1+\tau e_{1}-\sigma e_{2}\right), \frac{1}{2}\left(1-\tau e_{1}+\sigma e_{2}\right)\right\} \\
10_{+}^{\prime}= & \left\{\frac{1}{2}\left(1-e_{1}+e_{2}-e_{3}\right), \frac{1}{2}\left(1+e_{1}-e_{2}-e_{3}\right),\right. \\
& \frac{1}{2}\left(1-e_{1}+e_{2}+e_{3}\right), \frac{1}{2}\left(1+e_{1}-e_{2}-e_{3}\right), \\
& \left.\frac{1}{2}\left(1 \pm \tau e_{2} \pm \sigma e_{3}\right), \frac{1}{2}\left(1+\tau e_{1}+\sigma e_{2}\right), \frac{1}{2}\left(1-\tau e_{1}-\sigma e_{2}\right)\right\}
\end{aligned}
$$

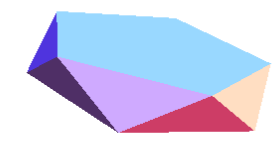

(a)

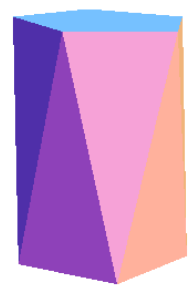

(b)

Figure 9: Distorted pentagonal antiprisms obtained from a dodecahedron (a) the solid represented by the set of vertices of $10_{+}$and (b) the solid represented by the vertices of $10_{+}^{\prime}$.

The intersection of the hyperplane orthogonal to the quaternion 1 with the sphere $S^{3}$ at the origin will lead to 20 vertices of the grand antiprism given by 


$$
\begin{aligned}
& \pm e_{1}, \pm e_{2}, \frac{1}{2}\left( \pm \sigma e_{1} \pm \tau e_{2} \pm e_{3}\right), \pm \frac{1}{2}\left(e_{1}+\sigma e_{2}+\tau e_{3}\right) \\
& \pm \frac{1}{2}\left(e_{1}+\sigma e_{2}-\tau e_{3}\right), \pm \frac{1}{2}\left(-\tau e_{1}+e_{2}+\sigma e_{3}\right), \pm \frac{1}{2}\left(\tau e_{1}-e_{2}+\sigma e_{3}\right)
\end{aligned}
$$

These are the remaining quaternions of the set 30 of Table 1 after removing 10 quaternions $\left\{e_{3} b^{m}\right\},(m=0,1, \ldots, 9)$.

Under the group $C_{2} \times W\left(H_{2}^{\prime}\right)$ the set of quaternions in (32) splits as

$$
\begin{aligned}
P_{1}= & \left\{ \pm e_{1}, \pm \frac{1}{2}\left(e_{1}+\sigma e_{2}+\tau e_{3}\right), \pm \frac{1}{2}\left(e_{1}+\sigma e_{2}-\tau e_{3}\right),\right. \\
& \left. \pm \frac{1}{2}\left(-\sigma e_{1}+\tau e_{2} \pm e_{3}\right)\right\}, \\
P_{2}= & \left\{ \pm e_{2}, \pm \frac{1}{2}\left(\tau e_{1}-e_{2}+\sigma e_{3}\right), \pm \frac{1}{2}\left(-\tau e_{1}+e_{2}+\sigma e_{3}\right),\right. \\
& \left. \pm \frac{1}{2}\left(\sigma e_{1}+\tau e_{2} \pm e_{3}\right)\right\}
\end{aligned}
$$

The two sets in (33) are pentagonal antiprism and non-regular stretched antiprism respectively as plotted in Figure 10.

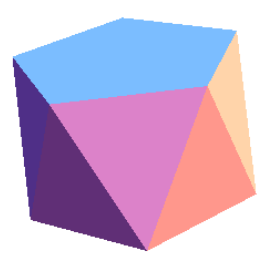

(a)

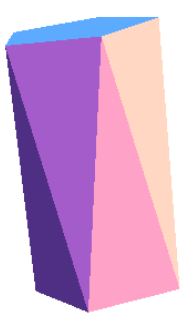

(b)

Figure 10: (a) Pentagonal antiprism represented by the vertices in (33) (first line), (b) The solid representing the vertices of (33) (second line)

\section{Dual of the grand antiprism}

To determine one cell of the dual grand antiprism one should find the centers of the cells which share one vertex of the grand antiprism. Let us choose a particular vertex represented by the quaternion $c$. We have seen that there are 2 pentagonal antiprisms and 12 tetrahedra sharing the quaternion $c$ as a common vertex. We have already noted that the centers of two pentagonal antiprisms are $\frac{\tau}{2} 1$ and $\frac{\tau}{2} b$. The centers of the 12 tetrahedra can be determined by averaging the corresponding vertices. The centers of the tetrahedra, up to a scale factor, are given by the following unit quaternions: 


$$
\begin{array}{ll}
c_{1}=\frac{1}{2 \sqrt{2}}\left(\tau+e_{1}+2 e_{2}-\sigma e_{3}\right), & c_{2}=\frac{1}{2 \sqrt{2}}\left(\tau-\sigma e_{1}+\sqrt{5} e_{2}\right), \\
c_{3}=\frac{1}{2 \sqrt{2}}\left(2-\sigma e_{1}+\tau e_{2}+e_{3}\right), & c_{4}=\frac{1}{2 \sqrt{2}}\left(2+\tau e_{1}+e_{2}+\sigma e_{3}\right), \\
c_{5}=\frac{1}{2 \sqrt{2}}\left(\sqrt{5}+\tau e_{1}-\sigma e_{2}\right), & c_{6}=\frac{1}{2 \sqrt{2}}\left(\sqrt{5}+e_{1}+e_{2}-e_{3}\right), \\
c_{7}=\frac{1}{2 \sqrt{2}},\left(\tau+\tau e_{1}+\tau e_{2}-\sigma^{2} e_{3}\right), & c_{8}=\frac{1}{2 \sqrt{2}}\left(\tau+e_{1}+2 e_{2}+\sigma e_{3}\right), \\
c_{9}=\frac{1}{2 \sqrt{2}}\left(\tau+\tau e_{1}+\tau e_{2}+\sigma^{2} e_{3}\right), & c_{10}=\frac{1}{2 \sqrt{2}}\left(2+\tau e_{1}+e_{2}-\sigma e_{3}\right), \\
c_{11}=\frac{1}{2 \sqrt{2}}\left(\sqrt{5}+e_{1}+e_{2}+e_{3}\right), & c_{12}=\frac{1}{2 \sqrt{2}}\left(2-\sigma e_{1}+\tau e_{2}-e_{3}\right) .
\end{array}
$$

One can easily check that any vector $c_{i}-c_{j},(i \neq j=1,2, \ldots, 12)$ is orthogonal to the vector $c$. One notes that the quaternion $1-b$ is also orthogonal to the quaternion $c$. However the quaternions $1-c_{i}$ and $b-c_{i}$ are not orthogonal to the quaternion $c$. The relative magnitudes of 1 and $b$ must be fixed to have their tips on the same hyperplane with the vectors $c_{i}$. One can easily check that the quaternions $c_{13}=\frac{\tau}{\sqrt{2}} 1$ and $c_{14}=\frac{\tau}{\sqrt{2}} b=\frac{1}{2 \sqrt{2}}\left(\tau+\sigma e_{1}+e_{2}\right)$ satisfy the requirement. These 14 quaternions are in a single hyperplane which is orthogonal to the vertex $c$. The 14 quaternions $c_{i},(i=1,2, \ldots, 14)$ represent the vertices of the cell of the dual grand antiprism. It is clear that the vertices of the dual grand antiprism have two different lengths, 20 of which are of length $\frac{\tau}{\sqrt{2}}$ and the remaining 300 vertices are of unit length. Therefore, vertices of the dual grand antiprism lie on two concentric spheres $S^{3}$ with radii $\frac{\tau}{\sqrt{2}}$ and 1 . The cell can be plotted, similar to the vertex figure, in the space of intersections of the hyperplane, orthogonal to the quaternion $c$, with two $S^{3}$ spheres. This means we need to use the basis introduced in (27). When 14 vertices $c_{i},(i=1,2, \ldots, 14)$, are expressed in terms of the new basis vectors then all first components would read $\frac{\tau^{2}}{2 \sqrt{2}} d_{0}$ and the remaining three components, up to a scale factor $\frac{1}{2 \sqrt{2}}$, can be written as

$$
\begin{array}{ll}
c_{1} \approx(-\sigma,-\sigma,-\sigma), & c_{2} \approx\left(0,1, \sigma^{2}\right), \\
c_{3} \approx\left(\sigma^{2}, 0,1\right), & c_{4} \approx\left(\sigma^{2}, 0,-1\right), \\
c_{5} \approx(-\sigma, \sigma, \sigma), & c_{6} \approx\left(-\sigma^{2}, 0,-1\right) \\
c_{7} \approx(-\sigma,-\sigma, \sigma), & c_{8} \approx\left(0,1,-\sigma^{2}\right), \\
c_{9} \approx\left(1, \sigma^{2}, 0\right) & c_{10} \approx\left(1,-\sigma^{2}, 0\right), \\
c_{11} \approx(-\sigma, \sigma,-\sigma), & c_{12} \approx(\sigma,-\sigma, \sigma), \\
c_{13} \approx(-1,-\tau, 0), & c_{14} \approx(-\tau, 0,1) .
\end{array}
$$

We obtain the following solid in three dimensions as shown in Figure 11. This cell has the same symmetry $C_{2} \times C_{2}$ of the vertex figure which fixes the quaternion $c$. The following sets of vertices of the cell are left invariant under the action of $C_{2} \times C_{2}$;

$$
\left(c_{1}, c_{4}, c_{8}, c_{10}\right),\left(c_{2}, c_{5}\right),\left(c_{3}, c_{6}, c_{11}, c_{12}\right),\left(c_{7}, c_{9}\right),\left(c_{13}, c_{14}\right)
$$




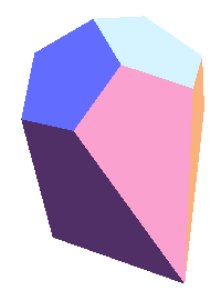

Figure 11: One cell of the dual grand antiprism

One cell of the dual grand antiprism consists of four regular pentagons, four kites and two isosceles trapezoids where the edge lengths are shown in Figure 12 .

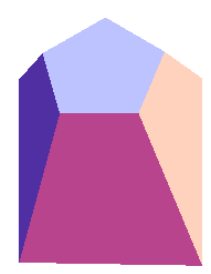

Figure 12: Faces of the cell of dual grand antiprism

To visualize the cell of the dual grand antisprism we note that the following sets of vertices form the faces of the cell:

Pentagons :

$\left(c_{1}, c_{2}, c_{8}, c_{7}, c_{9}\right), \quad\left(c_{8}, c_{12}, c_{6}, c_{4}, c_{7}\right)$,

$\left(c_{7}, c_{4}, c_{5}, c_{10}, c_{9}\right), \quad\left(c_{9}, c_{10}, c_{11}, c_{3}, c_{1}\right)$;

Kites :

$\left(c_{14}, c_{2}, c_{1}, c_{3}\right), \quad\left(c_{14}, c_{2}, c_{8}, c_{12}\right), \quad\left(c_{13}, c_{5}, c_{4}, c_{6}\right), \quad\left(c_{13}, c_{11}, c_{10}, c_{5}\right) ;$

Isosceles trapezoids :

$\left(c_{13}, c_{14}, c_{12}, c_{6}\right), \quad\left(c_{13}, c_{14}, c_{3}, c_{11}\right)$.

Let us note that the pair of vertices $\left(c_{13}, c_{14}\right) \in H_{2}$ is left invariant by a group larger than the group $C_{2} \times C_{2}$. Indeed the largest group fixing the set $\left(c_{13}, c_{14}\right) \in H_{2}$ is the direct product of two groups $C_{2} \times W\left(H_{2}^{\prime}\right)$ where the first group is generated by the group element $\left[\bar{b}^{2},-\bar{b}^{2}\right]^{*} \in W\left(H_{2}\right)$. The generators of the group $C_{2} \times W\left(H_{2}^{\prime}\right)$ can be chosen as follows 


$$
C_{2} \times W\left(H_{2}^{\prime}\right)=\left\{[\bar{b}, b],\left[e_{3},-e_{3}\right]^{*},\left[\bar{b}^{2},-\bar{b}^{2}\right]^{*}\right\} .
$$

The center of the cell of the dual grand antiprism represented by the vertex $c$, as we know, is left invariant by two generators in (38). The generator $[\bar{b}, b]$ generates the five vertices $\bar{b}^{m} c b^{m}, \quad(m=0,1,2,3,4)$ of the grand antiprism when applied to $c$. Action of the group element $[\bar{b}, b]$ on the other vertices $c_{i},(i=1,2, \ldots, 12)$ will generate other cells joined to the vertices $\left(c_{13}, c_{14}\right) \in$ $H_{2}$. That is to say we generate five cells all sharing the vertices $c_{13}$ and $c_{14}$. For each pair of quaternions $\left(b^{m}, b^{m+2}\right),(m=0,1, \ldots, 9)$ we have $5 \times 10=50$ cells overall and $\left(e_{3} b^{m}, e_{3} b^{m+2}\right),(m=0,1, \ldots, 9)$ another 50 cells with a total of 100 cells of the dual grand antiprism. This is of course the number of vertices of the grand antiprism. As we noted before, the dual grand antiprism has 20 vertices belonging to the root system $H_{2} \oplus H_{2}^{\prime}$. The remaining 300 vertices belong to two different orbits of $A u t\left(H_{2} \oplus H_{2}^{\prime}\right)$ of sizes 200 and 100 . They can be computed as follows. In the earlier paper [10] we determined the 600 vertices of the 120 -cell as the set of quaternions $J=\sum_{i, j=o}^{5} b^{i} T^{\prime} b^{j}$ where $T^{\prime}=V_{1} \oplus V_{2} \oplus V_{3}$ represents the vertices of the 24-cell with $V_{1}, V_{2}, V_{3}$ given by

$$
\begin{aligned}
& V_{1}=\left\{\frac{1}{\sqrt{2}}\left( \pm 1 \pm e_{1}\right), \frac{1}{\sqrt{2}}\left( \pm e_{2} \pm e_{3}\right)\right\} \\
& V_{2}=\left\{\frac{1}{\sqrt{2}}\left( \pm 1 \pm e_{2}\right), \frac{1}{\sqrt{2}}\left( \pm e_{3} \pm e_{1}\right)\right\} \\
& V_{3}=\left\{\frac{1}{\sqrt{2}}\left( \pm 1 \pm e_{3}\right), \frac{1}{\sqrt{2}}\left( \pm e_{1} \pm e_{2}\right)\right\}
\end{aligned}
$$

It is not difficult to see that the 600 vertices of the 120-cell decompose under the group $\operatorname{Aut}\left(H_{2} \oplus H_{2}^{\prime}\right)$ as $600=200+200+100+100$. With the choice of $b=\frac{1}{2}\left(\tau+\sigma e_{1}+e_{2}\right)$, we see that the 300 vertices of the dual grand antiprism are given by the set of quaternions

$$
\begin{aligned}
& J_{1}=\sum_{i, j=0}^{5} b^{i} V_{1} b^{j} \text { of size } 200 \\
& J_{3}^{\prime}=\sum_{i, j=0}^{5} b^{i} \frac{1}{\sqrt{2}}\left( \pm 1 \pm e_{3}\right) b^{j} \text { of size } 100 .
\end{aligned}
$$

One can check that in (35) the first 12 vertices belong to the set of 20 vertices of a dodecahedron and form four pentagonal faces only instead of 12 pentagonal faces of a dodecahedron. This is because 8 vertices are missing from the dodecahedron which is a typical cell of the 120-cell. For each vertex of the grand antiprism we have 12 vertices forming 4 pentagons which is part of the dual cell, then the number of vertices of the dual grand antiprism belonging to the set $\mathrm{J}$ is $\frac{100 \times 12}{4}=300$ as we have explained before which is the union of the sets $J_{1}$ and $J_{3}^{\prime}$. 


\section{Conclusion}

We have constructed the grand antiprism and its dual polytope with quaternions as a subset of the vertices of the 600-cell represented by the quaternionic elements of the binary icosahedral group. It is a $4 \mathrm{D}$ semi-regular polytope with 100 vertices and 320 cells made of 300 tetrahedral and 20 pentagonal antiprisms. Construction of its vertices and its symmetry group elements in terms of quaternions is very simple and very elegant. One needs only two orthogonal quaternions $b$ and $e_{3}$ to obtain the group elements and an additional quaternion $c$ for the construction of the vertices. The originality of the work lies in the fact that both the vertices of the grand antiprism and its symmetry group are constructed in terms of quaternions. We also note that the dual polytope of the grand antiprism has not been constructed elsewhere.

\section{Acknowledgement}

We would like to thank Dr. T. Yashiro and Dr. B. Anchouche for discussions. 


\section{Appendix: Decomposition of the semiregular orbits of $W\left(H_{4}\right)$ under the its maximal sub- group $\operatorname{Aut}\left(H_{2} \oplus H_{2}^{\prime}\right) \approx\left\{W\left(H_{2}\right) \times W\left(H_{2}^{\prime}\right)\right\}: C_{4}$}

Orbit 1:

$600=2(100)+2(200)$

Orbit 2:

$1200=3(200)+2(100)+400$.

Orbit 3:

$720=100+3(200)+20$.

Orbit 4:

$120=20+100$.

Orbit 5:

$3600=6(400)+5(200)+2(100)$.

Orbit 6:

$2400=6(200)+3(400)$.

Orbit 7:

$3600=5(200)+6(400)+2(100)$.

Orbit 8:

$1440=5(200)+400+40$.

Orbit 9:

$2400=3(400)+6(200)$.

Orbit 10:

$3600=6(400)+5(200)+2(100)$.

Orbit 11:

$7200=6(200)+15(400)$.

Orbit 12:

$7200=15(400)+6(200)$.

Orbit 13:

$7200=15(400)+6(200)$.

Orbit 14:

$7200=15(400)+6(200)$.

Orbit 15:

$14400=36(400)$. 


\section{References}

[1] M. Koca, R. Koc, M.Al-Barwani, J.M.Phys. 44 (2003) 03123; M. Koca, R. Koc, M. Al-Barwani, J. M. Phys 47 (2006) 043507-1; M.Koca, R. Koc, M. Al-Ajmi, J. Phys. A: Math. Gen. 39 (2006) 14047.

[2] H. Georgi and S.L. Glashow, Phys Rev. Lett. 32 ( 1974) 438.

[3] T. Pengpan and P.Ramond, Phys.Rep.C315 (1999) 137; P.Ramond, "Algebraic Dreams", UFIFT-HET-01-27.

[4] H. S. M. Coxeter, Regular Polytopes(3rd Edition), Dover Publications, INC. New York, 1973 ; H. S. M. Coxeter, Regular Complex Polytopes, Cambridge: Cambridge University Press, 1973.

[5] P. du Val, Homographies, Quaternions and Rotations, Oxford University Press, 1964;

[6] J.H. Conway and D. A. Smith, On Quaternions and Octonions: their geometry, arithmetic, and symmetry, A. K. Peters Ltd, Natic, Massachusetts.

[7] V. Elsver, N. J. A. Sloane, J. Phys. A 20 (1987) 6161; M. Koca, J. Phys.: Math.Gen. A22 (1989) 1949; ibid J.Phys. Math.Gen. A22 (1989) 4125; R.V. Moody and J. Patera, J Phys A: Math Gen. 26 (1993)2829.

[8] M. Koca, R. Koc, M. Al-Barwani,J.Phys.A 34 (2001)11201.

[9] J. H. Conway and Guy, Four-Dimensional Archimedean Polytopes, Proceedings of the Colloquium on Convexity at Copenhagen, page 38 und 39, 1965. ; http://en.wikipedia.org/wiki/Uniform-polycoron;

[10] M. Koca, R. Koc and M. Al- Ajmi, J. Phys. A: Math. Theor. 40 (2007) 7633 .

[11] M. Koca, R. Koc, M.Al-Barwani and S. Al-Farsi, Linear Algeb. Appl. 412 (2006) 441.

[12] M. Koca, M. Al-Ajmi and R. Koc, J. M. Phys., 48 (2007) 113514. 